\title{
MicroRNA-182 prevents vascular smooth muscle cell dedifferentiation via FGF9/PDGFRß signaling
}

\author{
NANA DONG ${ }^{1}$, WEI WANG ${ }^{1}$, JINWEI TIAN $^{1}$, ZULONG XIE $^{1}$, BO LV ${ }^{1}$, JIANNAN DAI $^{1}$, RUI JIANG ${ }^{1}$, \\ DAN HUANG $^{1}$, SHAOHONG FANG ${ }^{2}$, JIANGTIAN TIAN ${ }^{2},{\text { HULUN } L^{2} \text { and BO YU }}^{1,2}$ \\ ${ }^{1}$ Department of Cardiology, The Second Affiliated Hospital of Harbin Medical University; \\ ${ }^{2}$ Key Laboratory of Myocardial Ischemia, Ministry of Education, The Second Affiliated Hospital of \\ Harbin Medical University, Harbin, Heilongjiang 150086, P.R. China
}

Received December 3, 2015; Accepted January 20, 2017

DOI: $10.3892 /$ ijmm.2017.2905

\begin{abstract}
The abnormal phenotypic transformation of vascular smooth muscle cells (SMCs) causes various proliferative vascular diseases. MicroRNAs (miRNAs or miRs) have been established to play important roles in SMC biology and phenotypic modulation. This study revealed that the expression of miR-182 was markedly altered during rat vascular SMC phenotypic transformation in vitro. We aimed to investigate the role of miR-182 in the vascular SMC phenotypic switch and to determine the potential molecular mechanisms involved. The expression of miR-182 gene was significantly downregulated in cultured SMCs during dedifferentiation from a contractile to a synthetic phenotype. Conversely, the upregulation of miR-182 increased the expression of SMC-specific contractile genes, such as $\alpha$-smooth muscle actin, smooth muscle $22 \alpha$ and calponin. Additionally, miR-182 overexpression potently inhibited SMC proliferation and migration under both basal conditions and under platelet-derived growth factor-BB stimulation. Furthermore, we identified fibroblast growth factor 9 (FGF9) as the target gene of miR-182 for the phenotypic modulation of SMCs mediated through platelet-derived growth factor receptor $\beta$ (PDGFR $\beta$ ) signaling. These data suggest that miR-182 may be a novel SMC phenotypic marker and a modulator that may be used to prevent SMC dedifferentiation via FGF9/PDGFR $\beta$ signaling.
\end{abstract}

\section{Introduction}

Vascular smooth muscle cells (SMCs) retain remarkable plasticity to alternate from a differentiated to a dedifferentiated phenotype at different developmental stages or local environmental cues (1). The cellular switching process of SMCs from a quiescent contractile differentiated phenotype which is

Correspondence to: Professor Bo Yu, Department of Cardiology, The Second Affiliated Hospital of Harbin Medical University, 148 Baojian Road, Nangang, Harbin, Heilongjiang 150086, P.R. China

E-mail: dryu_hmu@163.com

Key words: vascular smooth muscle cells, microRNA-182, fibroblast growth factor 9 , platelet-derived growth factor receptor $\beta$ associated with the high expression of smooth muscle-specific marker genes, such as $\alpha$-smooth muscle actin (SMA), smooth muscle $22 \alpha(S M 22 \alpha)$ and calponin, to a synthetic dedifferentiated phenotype which is associated with decreased levels of the marker genes plays a critical role in many proliferative vascular diseases (1-3). This phenotypic switch is believed to be essential for vascular repair (3). However, for various cardiovascular diseases, the inhibition of abnormal switching and the control of SMC proliferation are critical therapeutic strategies.

MicroRNAs (miRNAs or miRs) are non-coding RNAs measuring $\sim 22$ nucleotides in length. They act as post-transcriptional negative repressors of protein expression by binding to the 3'-untranslated region (3'-UTR) of their target messenger RNAs (mRNAs) (4-6). miRNAs are known to play a critical role in cancer and cardiovascular disorders (7). Several miRNAs have emerged as important modulators of vascular SMC function and phenotype (8-10). The expression of miR-182 has been previously shown to be altered in various types of cancer, and it inhibits the proliferation and migration of rat Schwann cells (11-13).

Fibroblast growth factor (FGF) 9, a member of the FGF family, is a potent mitogen secreted from bone marrow cells $(14,15)$. FGF9 has been reported to be one of the direct targets of miR-182, which is associated with a variety of vessel biological processes $(13,16)$. Frontini et al revealed that FGF9 stimulates smooth muscle cells (SMCs) wrapping microvessels in implants required sonic hedgehog $(\mathrm{SHH})$ and platelet-derived growth factor (PDGF)R $\beta$ (16). PDGFs and their receptors (PDGFRs) are implicated in blood vessel pathophysiology (17). PDGFR $\beta$, expressed in perivascular mesenchyme, particularly in vascular mural cell (vascular SMCs and pericytes), has been established to play important roles in SMC differentiation and dedifferentiation $(17,18)$.

In this study, we found that miR-182 expression in cultured rat vascular SMCs was decreased with prolonged incubation (number of days) or with platelet-derived growth factor (PDGF)-BB treatment. Despite great advances in vascular SMC biology, the molecular mechanisms responsible for SMC phenotypic modulation remained unclear. Thus, the purpose of the study was to investigate the role of miR-182 in the vascular SMC phenotypic switch and to determine the underlying molecular mechanisms. 


\section{Materials and methods}

Rat vascular smooth muscle cell isolation. The animal protocols used were approved by the Scientific Affairs Committee of Animal Research and Ethics of the 2nd Hospital of Harbin Medical University. Vascular SMCs were isolated using the enzymatic dissociation method as previously described (19). Abdominal and thoracic aortic segments were isolated from Sprague-Dawley (SD) rats (weighting about $180 \mathrm{~g}$ ) under general anesthesia. All rats in this study were obtained from the Model Animal Center of Harbin Medical University. After removing the connective tissue, adventitia and the endothelial layer, aortas were sliced into 1-2-mm-thick fragments and incubated with Dulbecco's modified Eagle's medium (DMEM)/ F12 with the addition of type 2 collagenase $(1.4 \mathrm{mg} / \mathrm{ml})$ (Sigma, St. Louis, MO, USA) for $4-6 \mathrm{~h}$ in a $37^{\circ} \mathrm{C}$ incubator with $95 \%$ air and $5 \% \mathrm{CO}_{2}$. The fragments were agitated to release SMCs and the cells were centrifuged at $300 \mathrm{xg}$ for $5 \mathrm{~min}$. SMCs were then cultured in DMEM/F12 medium added with 10\% fetal bovine serum in an incubator as described above at least for 5 days prior to the first time trypsin digestion. The primary cultured SMCs were then passed every 3 days, and the 4-6 passages were used. In addition, SMCs released from the arterial fragments (0 days), cultured SMCs at 5 day, 10 and 15 days were also collected for examination.

Transfection of cultured SMCs with oligonucleotides. Oligonucleotide (oligo; Bioneer Co., Ltd., Daejeon, Korea) transfection was based on the instruction of Roche X-tremeGENE siRNA Transfection Reagent (Roche, Mannheim, Germany). For miR-182 overexpression or silencing, miR-182 mimics or inhibitors were added directly to the complexes to different final concentrations (miR-182 mimic: sense, GCG GGU CUA GCU GCC GGA and antisense, CGG CAG CUA GAC CCG CUU; miR-182 inhibitor: UUU GGC AAU GGU AGA ACU CAC ACC G). A small interfering RNA (siRNA) was used to degrade the target mRNAs for fibroblast growth factor 9(FGF9) gene silencing as previously described (sense, CUU CCA ACC UGU ACA AGA and antisense, UGC UUG UAC AGG UUG GAA G) (20). Briefly, following trypsin digestion, SMCs were inoculated in a 6-well plate. Twenty-four hours later, the $\mathrm{X}$-tremeGENE siRNA transfection reagent was used to treat the cells in a 1:4 ratio for $20 \mathrm{~min}$. Further treatment included transfection with siRNA followed by incubation in medium comprising $2 \mathrm{ml}$ of serum-free Opti-MEMI (Invitrogen, Carlsbad, CA, USA) using transfection reagents (vehicle), negative control, inhibitor negative control (Bioneer Co., Ltd.), and siRNA control (Invitrogen). The transfection efficiency of miR-182 mimic and inhibitor was then examined by reverse transcription-quantitative PCR (RT-qPCR) and the efficiency of FGF9 siRNA was examined by western blot analysis, as described below. Forty-eight hours later, the engineered SMCs were analyzed further.

miRNA and $m R N A$ analysis by $R T-q P C R$. Following oligo transfection and stimulation with or without PDGF-BB, total RNA was extracted from the SMCs using TRIzol reagent (15596-026; Invitrogen) according to the instructions of the manufacturer. After the abdominal and thoracic aortic segments were isolated from the anesthetized rats, organs including aorta, muscle, heart, lung, liver and kidney were also removed. Total RNA was then extracted from these organs based on the instructions of the manufaturer of TRIzol reagent. RNA was then reverse transcribed into cDNA using the First Stand miRcute miRNA cDNA Synthesis kit according to the instructions of the manufacturer (Tiangen Biotech, Beijing, China). The miR-182 level was analyzed by quantitative polymerase chain reaction (qPCR) using the Tiangen miRcute miRNA qPCR detection system (Poly A tail addition method; miR-182 primer, TTT GGC AAT GGT AGA ACT CAC ACC; U6 primer sequence, ACA CGC AAA TTC GTG AAG CGT TCC). The RT-qPCR for FGF9, plateletderived growth factor receptor $\beta$ (PDGFR $\beta$ ), SMA, SM $22 \alpha$ and calponin was performed using the Bioneer AccuPower GreenStar qPCR PreMix system (SMA forward, GTC AGG TCA TCA CTA TCG GCA AT and reverse, AGA GGT CTT TAC GGA TGT CAA CGT; SM22 $\alpha$ forward, ATG GCC AAC AAG GGT CCA TCC and reverse, TCC ATC TGC TTG AAG ACC ATG; calponin forward, AGAGAA GGC AGG AAC ATC ATT GGC and reverse, GTG TCA CAG TGT TCC ATG CCC AG; FGF9 forward, ACA GGA GTG CGT GTT CAG AG and reverse, GTT CAG GTA CTT TGT CAG GGT C; PDGFR $\beta$ forward, AGG ACA ACC GTA CCT TGG GTG ACT and reverse, CAG TTC TGA CAC GTA CCG GGT CTC). The expression of miR-182 relative to U6 and SMA, SM22 $\alpha$, calponin, FGF9, and PDGFR $\beta$ relative to $\beta$-actin was determined by the $2^{-\Delta \Delta C t}$ method.

Western blot analysis. Cellular proteins were extracted for western blot analysis as previously described (20). The cell lysates were subjected to sodium dodecyl sulfate polyacrylamide gel electrophoresis (SDS-PAGE) and run under standard conditions. Subsequently, the proteins were removed to polyvinylidene fluoride (PVDF) membranes. After blocking with 5\% nonfat milk at room temperature for $1 \mathrm{~h}$, the PVDF membranes were incubated with diluted primary antibodies SMA (1:400 dilution; sc-53142), SM22 $\alpha$ (1:300 dilution; sc-51442) (both from Santa Cruz Biotechnology, Santa Cruz, CA, USA), FGF9 (1:800 dilution; ab9743), PDGFR $\beta$ (1:1,000 dilution; ab32570) (both from Abcam, Cambridge, MA, USA), and antibody against GAPDH (1:1,000 dilution; sc-47724; Santa Cruz Biotechnology) at $4^{\circ} \mathrm{C}$ overnight. The membranes were washed, further incubated for $1 \mathrm{~h}$ with horseradish peroxidaseconjugated secondary antibodies (anti-mouse and anti-rabbit, ZB-2305 and ZB-2305; both from Zhongshan Goldenbridge Biotechnology, Beijing, China) at $37^{\circ} \mathrm{C}$. Subsequently, the membranes were detected with BeyoECL Plus (Beyotime Institute of Biotechnology, Haimen, China), and further analysis of protein band densitometry was facilitated by Quantity One software (Bio-Rad, Hercules, CA, USA).

SMC proliferation assay. SMCs at the 4th to 6 th passages were trypsinized and plated into 96 -well culture plates $\left(5 \times 10^{3}\right.$ cells/well) in DMEM/F-12 complete medium. After $24 \mathrm{~h}$, the SMCs were rendered quiescent in DMEM/F-12 supplemented with $0.5 \%$ FCS for $24 \mathrm{~h}$. The proliferation of the SMCs was examined by 3-(4,5-dimethylthiazol-2-yl)-2,5-diphenyltetrazolium bromide (MTT) assay (Solarbio, Beijing, China) or by 5-ethynyl2'-deoxyuridine (EdU) proliferation assay (Ribo-Bio Co., Ltd., Guangzhou, China). MTT assays were conducted as previously described $(8,9)$. In brief, the SMCs in each well were incubated with $20 \mu \mathrm{l}$ MTT mixture $(5 \mathrm{mg} / \mu \mathrm{l})$. Four hours later, formazan 

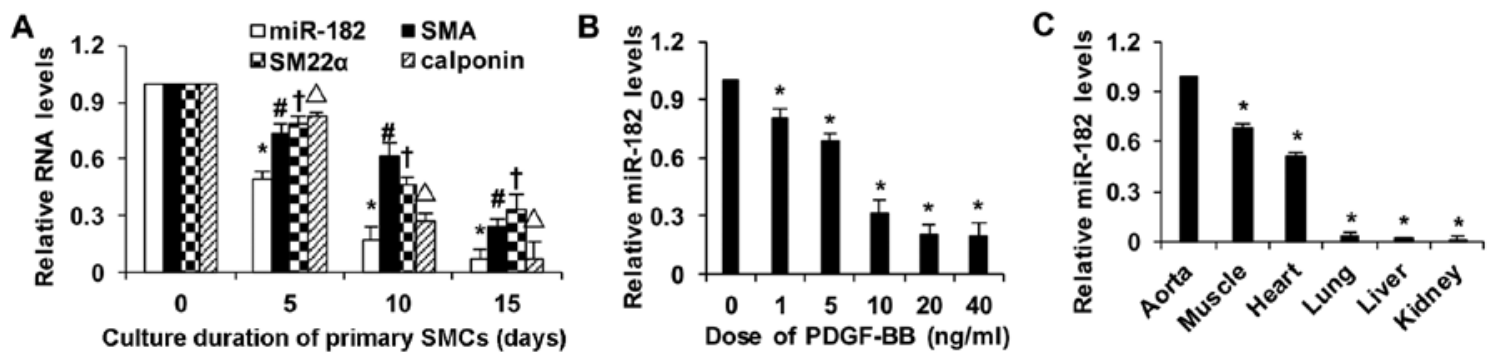

Figure 1. MicroRNA-182 (miR-182) is a novel marker for in rat vascular smooth muscle cell (SMC) phenotypic modulation. (A) Expression levels of differentiation marker genes [ $\alpha$-smooth muscle actin (SMA), smooth muscle actin (SM22 $\alpha$ ) and calponin] and miR-182 eventually declined with the increased duration of SMC culture, as determined by RT-qPCR ( $=5$ repeated experiments). ${ }^{*, \#, t, \Delta} \mathrm{p}<0.05$ compared with their corresponding data at 0 day; (B) Following treatment platelet-derived growth factor-BB (PDGF-BB) for $24 \mathrm{~h}$, miR-182 expression decreased in a dose-dependent manner. The levels of miR-182 did not differ significantly in SMCs stimulated by PDGF-BB at 20 and $40 \mathrm{ng} / \mathrm{ml}$. " p $<0.05$ vs. control group, as determined by qPCR (n=5); (C) miR-182 was expressed more highly in the aorta, as shown by RT-qPCR $(n=3) .{ }^{*} \mathrm{p}<0.05$ vs. the aorta.

crystals were accumulated in live cells. Subsequently, $100 \mu \mathrm{l}$ dimethyl sulphoxide (DMSO) were added to each well to solubilize the formazan crystals with 10 min shaking. The results of MTT assay were analyzed by optical density measurement at OD490 nm. EdU proliferation assays were conducted according to the manufacturer's instructions, for quantitative synthesis of DNA via the incorporation of a deoxyribonucleoside analog containing a detectable tag. Briefly, with SMCs, with the addition of EdU at a final concentration of $50 \mu \mathrm{M}$, were incubated for a further $2 \mathrm{~h}$. The cells were then fixed with $4 \%$ paraformaldehyde (PFA) for $30 \mathrm{~min}$, and permeabilized with PBS containing $0.5 \%$ Triton X-100 for $10 \mathrm{~min}$. Subsequently, SMCs were stained with 1X Apollo ${ }^{\circledR}$ reaction cocktail for $30 \mathrm{~min}$, and with Hoechst 33342 for $30 \mathrm{~min}$. The number of Hoechst 33342-stained cells in 10 random viewing fields were counted for analysis. The images were acquired using a fluorescence microscope (Leica DMI4000B; Leica, Solms, Germany).

SMC migration assay. Migration assays were performed by scratch wound healing as previously described (10). SMCs were pre-transfected with oligos, and plated into 6-well cultured plates ( $2.5 \times 10^{5}$ cells/well). SMCs, after being quiescent for $24 \mathrm{~h}$, were scratched using a $200 \mu \mathrm{l}$ disposable pipette tip to make a single scratch wound by streaking across a monolayer of cells. The SMCs were then treated with or without PDGF-BB (P-4056; Sigma). Images were captured at various times post-wounding using an Olympus IX 73 microscope equipped with a diagnostic instrument RT SPOT Digital Camera (Olympus, Tokyo, Japan). The migrated cells of each high-power field (x400) were counted to represent SMC migration.

Statistical analysis. The data were analyzed using IBM SPSS software (version 17.0; SPSS, Inc., Chicago, IL, USA). The data, averaged of at least three repeated experiments, are expressed as mean \pm standard deviation (SD). The relative gene expression was determined based on the mean value of the vehicle control group defined as $100 \%$. Data analysis was accomplished using twotailed unpaired Student's t-tests and ANOVA. A p-value <0.05 was considered to indicate a statistically significant difference.

\section{Results}

miR-182 is a novel marker for rat vascular SMC phenotypic modulation. The levels of the differentiation marker genes, including SMA, SM22 $\alpha$ and calponin, declined eventually with the prolonged incubation of rat vascular SMCs in vitro (Fig. 1A). During the phonotypic switch, the biosynthesis of miR-182 was also gradually diminished. On the 5th day of culture, the level of miR-182 in the SMCs decreased to almost half that of the level at 0 days. To examine the implications of this regulation, the SMCs were treated with various concentrations of PDGFBB, a powerful stimulant of SMC dedifferentiation. Treatment with PDGF-BB induced a decrease in miR-182 expression in a dose-dependent manner; treatment with $10 \mathrm{ng} / \mathrm{ml}$ PDGF-BB markedly reduced miR-182 expression; the expression levels of miR-182 did not differ significantly between the SMCs stimulated with PDGF-BB 20 and 40 ng/ml (Fig. 1B). Furthermore, the miR-182 distribution levels in the aorta, heart, muscle, lung, liver and kidney were examined by RT-qPCR. The expression level of miR-182 in the aorta was higher than that in other tissues (Fig. 1C). Taken together, these findings suggest that miR-182 may be a novel marker for SMC phenotypic modulation.

miR-182 is a novel phenotypic modulator of vascular SMCs. To explore the potential effect of miR-182 during SMC phenotypic modulation, gain-of-function and loss-of-function experiments were performed to transfect oligos into rat vascular SMCs. For miR-182 overexpression, we added miR-182 mimics at a final concentration of $20,40,60$ or $80 \mathrm{nM}$ to culture medium. miR-182 expression was increased depending on the oligo concentrations (Fig. 2A). Conversely, miR-182 expression was markedly inhibited by transfection with $80 \mathrm{nM}$ miR-182 inhibitors. Additionally, the gene levels of SMC-specific markers, including SMA, SM22 $\alpha$ and calponin, were distinctly increased by transfection with miR-182 mimics, and the marker gene levels were significantly decreased by transfection with miR-182 inhibitors (Fig. 2B). Similarly, as shown in Fig. 2C, the results of western blot analysis revealed that the transfection of SMCs with miR-182 mimic or inhibitor altered the protein levels of SMA and SM22 $\alpha$.

Subsequently, the biological role of miR-182 in primary cultured rat vascular SMC morphology was investigated. The primary cultured SMCs at passages 4 to 6 transfected with miRNA negative control presented a flattened morphology as a synthetic phenotype (Fig. 2D). The overexpression of miR-182 altered SMC morphology from a flattened to a spindle-like contractile state. The cells flattened progressively when transfected with miR-182 inhibitors or treated with PDGF-BB (10 ng/ 

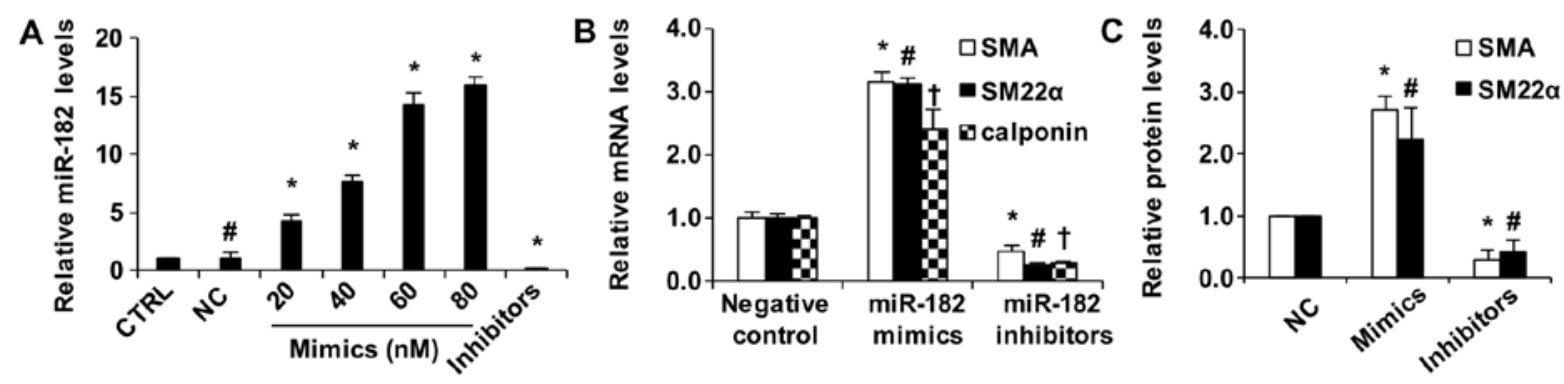

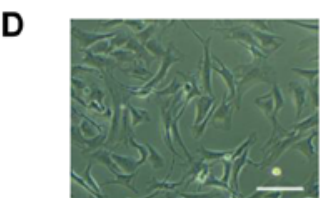

Negative control

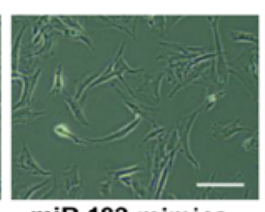

miR-182 mimics

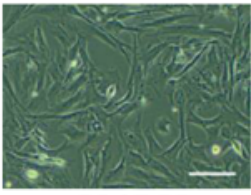

PDGF-BB

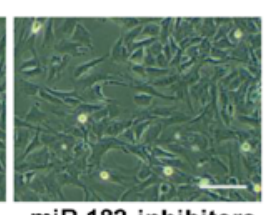

miR-182 inhibitors

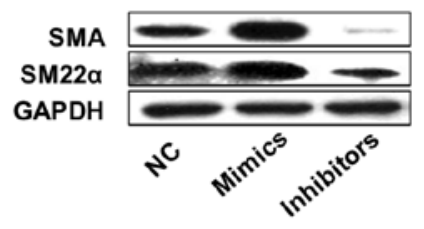

Figure 2. Role of miR-182 in the phenotypic modulation of rat vascular smooth muscle cells (SMCs). (A) miRNA-negative control (NC), miR-182 mimics (20, $40,60$ and $80 \mathrm{nM})$ or inhibitors $(80 \mathrm{nM})$ were transfected into rat vascular SMCs. Intracellular miR-182 expression was significantly increased depending on the miR-182 mimic concentrations, as determined by RT-qPCR ( $\mathrm{n}=5)$. ${ }^{*} \mathrm{p}<0.05$ compared with the control (CTRL). ${ }^{*} \mathrm{p}<0.05 \mathrm{vs}$. NC. (B) The effect of miR-182 mimics $(80 \mathrm{nM})$ and miR-182 inhibitors $(80 \mathrm{nM})$ on the expression of SMA, smooth muscle $22 \alpha(\mathrm{SM} 22 \alpha)$, and calponin, as determined by RT-qPCR ( $=4)$. ${ }^{*} \mathrm{p}<0.05$ vs. NC group of the SMA expression; ${ }^{\#} \mathrm{p}<0.05$ vs. NC group of the SM22 $\alpha$; ${ }^{\dagger} \mathrm{p}<0.05$ vs. NC group of the calponin. (C) The effect of miR-182 mimics $(80 \mathrm{nM})$ and miR-182 inhibitors $(80 \mathrm{nM})$ on the protein expression of SMA and SM22 $\alpha$, as determined by western blot analysis (n=4). *p<0.05 vs. NC group of SMA; ${ }^{\#} \mathrm{p}<0.05$ vs. NC group of SM22 $\alpha$. (D) Morphology of primary cultured rat vascular SMCs. SMCs were treated with miR-182 mimics $80 \mathrm{nM}$, inhibitors $80 \mathrm{nM}$ or PDGF-BB (10 ng/ml). Magnification, x400. Scale bars, $50 \mu \mathrm{m}$.

ml). miR-182 overexpression helped the SMCs retain their contractile morphology and prevented the phenotypic switch. On the whole, these findings suggest that miR-182 is essential in modulating the rat vascular SMC phenotype.

miR-182 inhibits rat vascular SMC proliferation and migration. Typical images of EdU cell proliferation are shown in Fig. 3A. miR-182 overexpression markedly suppressed SMC proliferation even with PDGF-BB $(5 \mathrm{ng} / \mathrm{ml})$ treatment in vitro. In addition, PDGF-BB treatment increased SMC proliferation, which was further enhanced by transfection with miR-182 inhibitors. MTT assay was also performed to determine the mitochondrial activity. Transfection with miR-182 mimics at $80 \mathrm{nM}$ significantly inhibited cell proliferation to a greater extent than at 20 and $40 \mathrm{nM}$ (Fig. 3B).

Furthermore, we examined the effect of miR-182 on SMC migration in vitro by scratch-wound healing assay. The migration of SMCs transfected with miR-182 mimics was markedly inhibited even with PDGF-BB stimulation (Fig. 3C and D). However, transfection with miR-182 inhibitors increased the PDGF-BB-induced migration of SMCs.

To the best of our knowledge, for the first time, in this study, we established that miR-182 effectively suppressed the proliferation and migration of rat vascular SMCs under both quiescent conditions and PDGF-BB stimulation.

miR-182 prevents SMC phenotypic modulation via FGF9/ PDGFR $\beta$ signaling. miRNAs exert their biological functions by inhibiting the transcription or translation of their target genes. We used the microRNA.org database to identify the target genes of miR-182. FGF9 is a target gene of miR-182 across multiple species, such as human, rat and mouse (Fig. 4A). Using qPCR, we discovered that transfection with miR-182 mimic or inhibitor slightly inhibited or increased the FGF9 mRNA levels, respectively (Fig. 4B). However, using western blot analysis, the protein levels of FGF9 were significantly affected by miR-182 regulation. The FGF9 levels were decreased when the SMCs were transfected with miR-182 mimics at $80 \mathrm{nM}$ more than at $40 \mathrm{nM}$ (Fig. 4C). On the contrary, the protein level of FGF9 was increased by transfection with miR-182 inhibitors at $80 \mathrm{nM}$ more than $40 \mathrm{nM}$ (Fig. 4D). These data suggest that FGF9 is the direct target gene of miR-182 in rat vascular SMCs.

To determine the role of FGF9 in rat SMCs, we used FGF9 siRNA transfection to achieve FGF9 downregulation. The protein levels of the SMC-specific contractile markers, SMA and SM22 $\alpha$, were significantly upregulated by FGF9 siRNA transfection (Fig. 5A). The protein levels of PDGFR $\beta$ were decreased with the knockdown of FGF9. Additionally, as shown in Fig. 5B and C, the knockdown of FGF9 significantly inhibited SMC proliferation with or without PDGF-BB stimulation, as determined by MTT and EdU assays. Moreover, when the SMCs were transfected with FGF9 siRNA, the migration induced by PDGF-BB was also significantly depressed (Fig. 5D and E). Therefore, the silencing of FGF9 suppressed the differentiation, proliferation and migration of rat SMCs.

Subsequently, miR-182 inhibitors and FGF9 siRNA were both transfected into the SMCs. The downregulation of miR-182 increased SMC proliferation and migration, and decreased the expression of dedifferentiated marker genes (Fig. 6A-C). However, FGF9 knockdown interfered with the function of miR-182 inhibitors on SMC proliferation and migration, but induced an increase in the expression of SMA, SM22 $\alpha$ and calponin. Overall, our data indicate that FGF9 is a direct target gene of miR-182 in rat vascular SMCs, and is critical in the process of miR-182-mediated rat vascular SMC phenotypic modulation.

We hypothesized that the inhibition of SMC phenotypic modulation via the upregulation of miR-182 or the downregulation of FGF9 was related to PDGFR $\beta$ signaling. PDGF-BB 


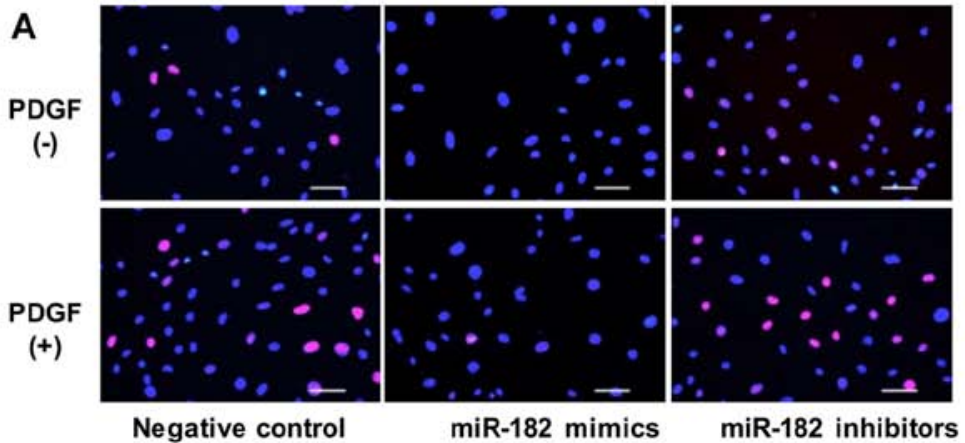

C

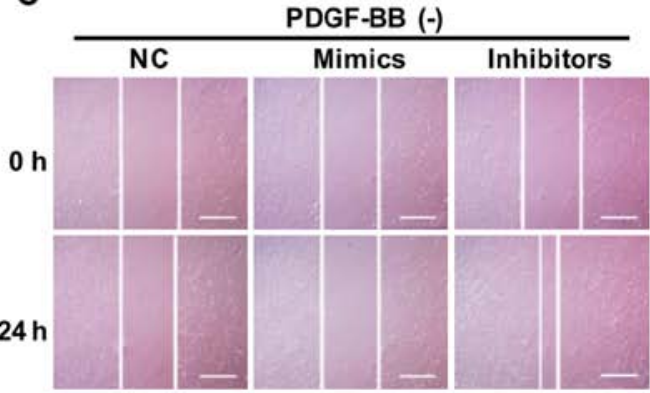

B

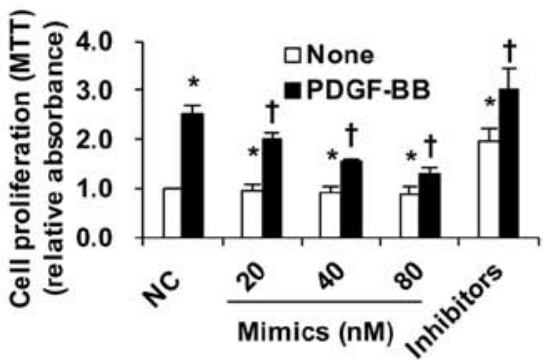

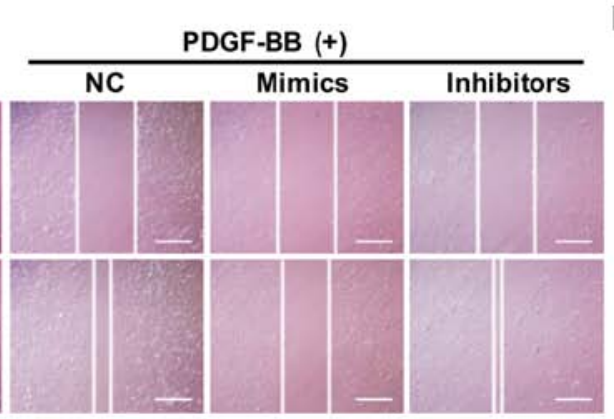

D

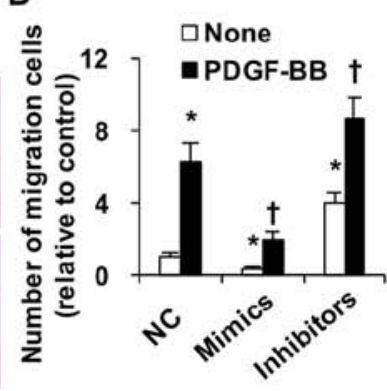

Figure 3. The role of miR-182 in rat vascular smooth muscle cells (SMCs) proliferation and migration. (A) EdU assay to evaluate proliferation in response to PDGF-BB $(5 \mathrm{ng} / \mathrm{ml})$ of SMCs transfected with miR-182 mimics $(80 \mathrm{nM})$ or inhibitors $(80 \mathrm{nM})$. The proliferative cells appear pink, while the non-proliferative cells are blue in color (nuclei stained with DAPI). Scale bars, $50 \mu \mathrm{m}$. (B) The proliferation was also detected by MTT assay (n=5). Transfection with miR-182 mimics at $80 \mathrm{nM}$ inhibited the proliferation induced-by PDGF-BB $(5 \mathrm{ng} / \mathrm{ml})$ more than at 20 and $40 \mathrm{nM}$. miR-182 inhibitors $(80 \mathrm{nM})$ transfection further enhanced PDGF-BB-induced proliferation of SMCs. " $\mathrm{p}<0.05$ vs. negative control (NC) without PDGF-BB treatment; ${ }^{\dagger} \mathrm{p}<0.05$ vs. NC with PDGF-BB treatment. (C) The typical pictures of Scratch-wound healing assay. SMCs were transfected with miR-182 mimics $(80 \mathrm{nM})$ or inhibitors $(80 \mathrm{nM})$. The 24-h migration of transfected cells with or without PDGF-BB $(5 \mathrm{ng} / \mathrm{ml})$ treatment was numbered. Scale bars, $50 \mu \mathrm{m}$. (D) Quantification of migrated SMCs. Data of the number of migrated cells relative to the NC group from 3 independent experiments are presented as means $\pm \mathrm{SD}(\mathrm{n}=5)$. ${ }^{\mathrm{p}} \mathrm{p}<0.05$ vs. NC without PDGF-BB treatment; ${ }^{\dagger} \mathrm{p}<0.05$ vs. NC with PDGF-BB treatment.

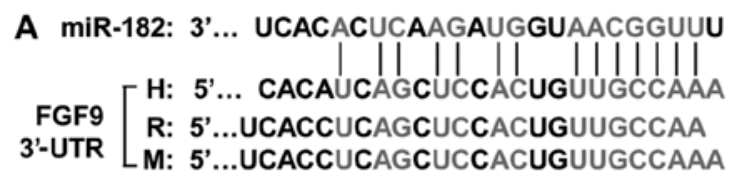

B

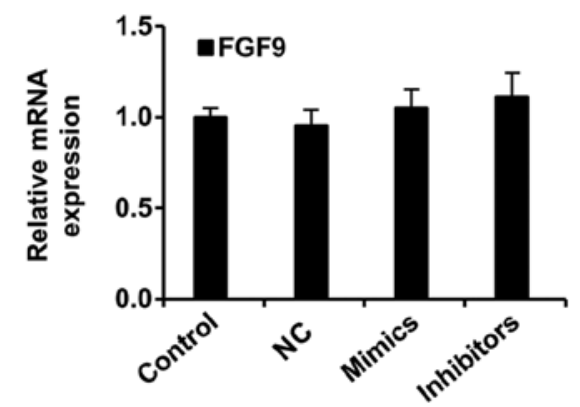

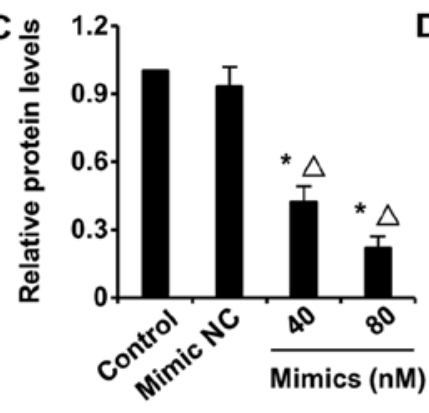
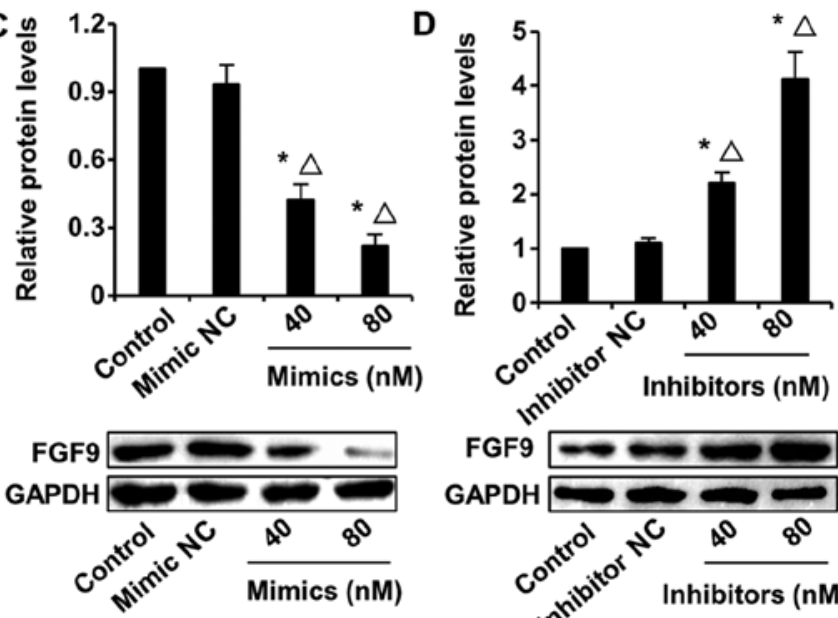

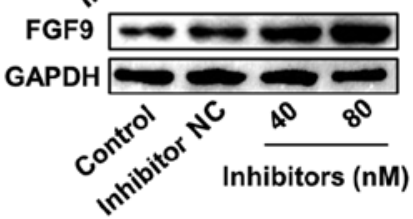

Figure 4. FGF9 is the target gene of miR-182 in rat vascular smooth muscle cells (SMCs). (A) The predicted binding sequences of miR-182 in FGF9 3'-UTR of human $(\mathrm{H})$, rat (R) and mouse (M). UTR, untranslated region. (B) As determined by RT-qPCR, the gene expression of FGF9 was not significantly altered by miR-182 mimic or inhibitor transfection in cultured SMCs $(n=5)$. NC, negative control. (C) Western blot and densitometric analyses revealed that the protein levels of FGF9 were decreased when SMCs were trasfected with miR-182 mimics ( $\mathrm{n}=5$ ). ${ }^{*} \mathrm{p}<0.05$ vs. control; ${ }^{\wedge} \mathrm{p}<0.05$ vs. mimic NC. (D) FGF9 protein was up-expressed with miR-182 inhibitor transfection, as determined by western blot analysis ( $\mathrm{n}=5)$. ${ }^{*} \mathrm{p}<0.05$ vs. control; ${ }^{\wedge} \mathrm{p}<0.05$ vs. inhibitor $\mathrm{NC}$.

induced an increase in PDGFR $\beta$ gene expression, while transfection with miR-182 mimics significantly inhibited PDGFR $\beta$ expression with or without PDGF-BB stimulation (Fig. 6D). Conversely, the gene levels of PDGFR $\beta$ were upregulated following transfection with miR-182 inhibitors. PDGF-BBinduced a significant increase in the PDGFR $\beta$ protein level, while miR-182 overexpression markedly suppressed PDGFR $\beta$ expression (Fig. 6E and F). In addition, transfection with FGF9 siRNA decreased PDGFR $\beta$ expression (Fig. 5A). Thus, these data indicate that FGF9/PDGFR $\beta$ signaling is critical for the miR-182-mediated prevention of the SMC phenotypic switch. 

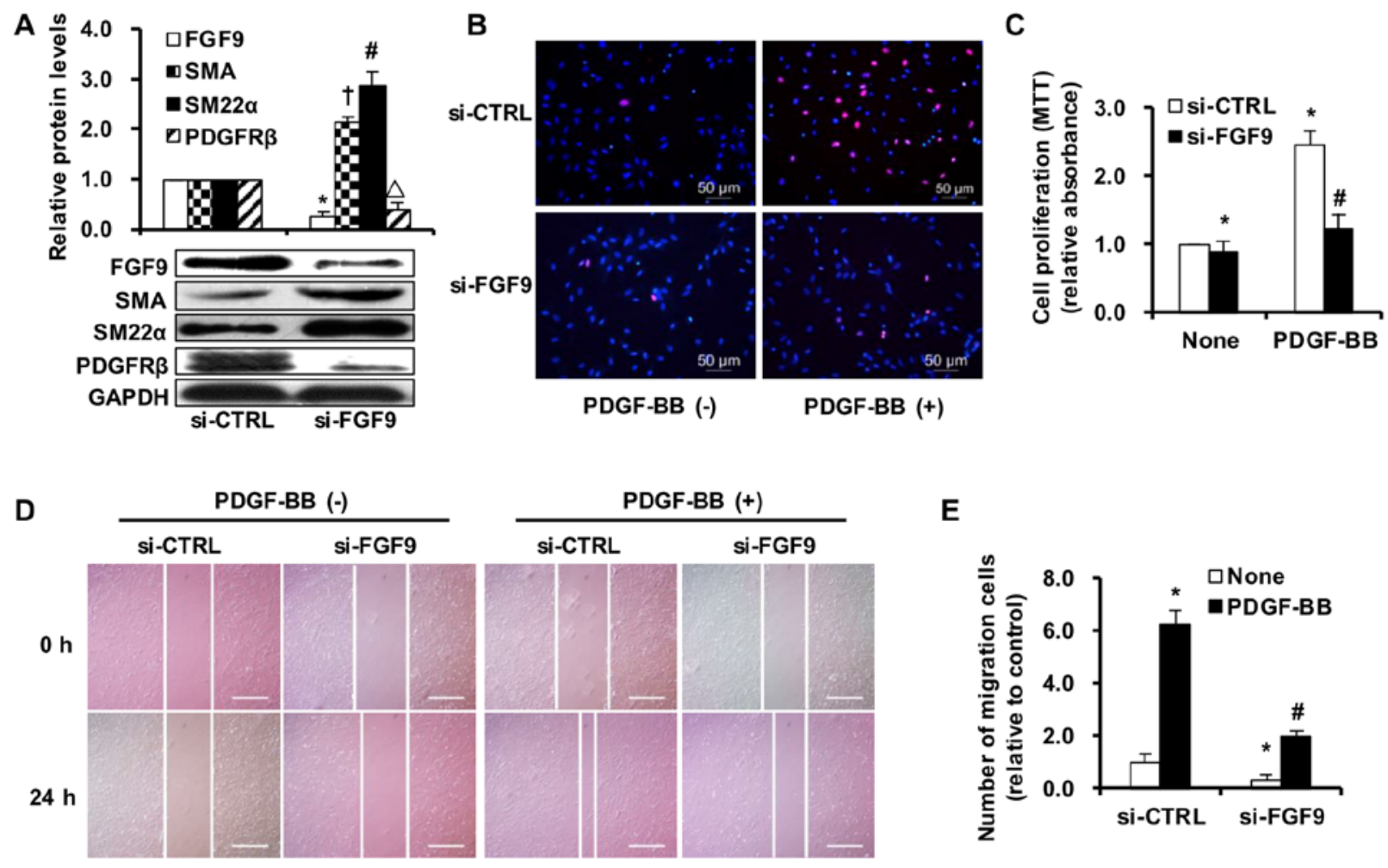

Figure 5. FGF9 plays an important role in the differentiation, proliferation and migration of rat smooth muscle cells (SMCs). (A) The protein levels of FGF9 in SMCs were markedly inhibited by transfection with FGF9 siRNA (100 nM) compared with siRNA control (si-CTRL, $100 \mathrm{nM})$ transfection (n=5; ${ }^{*} \mathrm{p}<0.05$ ). With FGF9 knockdown, the protein levels of SMA and smooth muscle $22 \alpha$ (SM22 $\alpha$ ) were upregulated, but the level of platelet-derived growth factor receptor $\beta$ (PDGFR $\beta$ ) was downregulated as determined by western blot and densitometric analyses $(\mathrm{n}=5)$. ${ }^{\dagger} \mathrm{p}<0.05$ vs. si-CTRL of SMA; ${ }^{*} \mathrm{p}<0.05$ vs. si-CTRL of SM22 $\alpha$; ${ }^{\triangle} \mathrm{p}<0.05$ vs. Si-CTRL of PDGFR $\beta$; (B and C) Knockdown of FGF9 significantly inhibited SMC proliferation with or without PDGF-BB stimulation, as determined by EdU [(B) magnification, $\mathrm{x} 400$. Scale bars, $50 \mu \mathrm{m}]$ and MTT assays [(C) $\mathrm{n}=5 .{ }^{*} \mathrm{p}<0.05$ vs. si-CTRL without PDGF-BB treatment; ${ }^{*} \mathrm{p}<0.05 \mathrm{vs}$. si-CRTL with PDGF-BB treatment] assays. (D) Typical images of Scratch-wound healing assay (scale bars, $50 \mu \mathrm{m}$ ). The migration of SMCs was significantly suppressed by si-FGF9 transfection, even with PDGF-BB stimulation. (E) Quantification of migrated SMCs. Data of the number of migrated cells relative to si-CTRL without PDGF-BB stimulated group are presented as the means $\pm \mathrm{SD}(\mathrm{n}=5)$, which was asseessed by 3 independent experiments. ${ }^{*}<<0.05$ vs. si-CTRL without PDGF-BB treatment; ${ }^{*} \mathrm{p}<0.05$ vs. si-CRTL with PDGF-BB treatment.

\section{Discussion}

In the present study, to the very best of our knowledge, we demonstrate for the first time miR-182 is a novel marker which has the capacity to modulate the rat vascular SMC phenotype. The critical target gene of miR-182 is FGF9, and miR-182-mediates the differentiation, proliferation and migration of rat vascular SMCs via FGF9/PDGFR $\beta$ signaling.

Despite great advances in vascular SMC biology, the molecular mechanisms responsible for the SMC phenotypic modulation remain unclear. miR-182 has been reported to exhibit an altered expression in various types of cancers, which may be a useful prognostic biomarker for cancer (21). Previous studies have revealed that miR-182 may function as an oncogenic miRNA to enhance cancer cell proliferation, aggressiveness, tumorigenesis and drug resistance (22-25). However, other studies have reported conflicting results, showing that miR-182 suppressed cell growth and aggression, and increased miR-182 levels were shown to correlate with clinical treatment benefits $(13,26-28)$. These results suggest the existence of highly complex mechanisms linking miR-182, cell growth and motility. In this study, we thus investigated the association between miR-182 expression and the phenotype of vascular SMCs. In this study, the expression of SMC-specific genes in rat vascular SMCs declined eventually with prolonged incubation, which was consistent with the find- ings of previous studies that dispersed SMCs cultured in vitro exhibited rapidly downregulated SMC-specific gene expression $(29,30)$. During the period that the SMC phenotype switches from a contractile to a synthetic type, the expression of miR-182 was also demonstrated to gradually decrease. Additionally, the miR-182 levels significantly decreased with PDGF-BB stimulation in a dose-dependent manner. For the first time, to the best of our knowledge, we established that miR-182 is a novel phenotypic marker for SMCs.

Increasing evidence indicates the important roles of miR-182 in cell biology. miR-182 has been shown to predict survival in patients with various types of cancer (21). Kouri et al found that miR-182 targeted Bcl-2-like 12, c-Met and hypoxia-inducible factor $2 \alpha$ to regulate the apoptosis, growth and differentiation programs of glioma-initiating cells (31). miR-182 has also been shown to inhibit Schwann cell proliferation and invasion following nerve injury and repair (13). In the present study, the overexpression of miR-182 inhibited the dedifferentiation of cultured vascular SMCs, as well as SMC proliferation and migration. Additionally, miR-182 overexpression prevented the SMCs from switching from a contractile morphology to a synthetic phenotype. Thus, miR-182 is a novel phenotypic modulator of SMC differentiation, proliferation and migration.

miRNAs exert their biological functions by inhibiting the transcription or translation of target genes $(4,6)$. By using the 

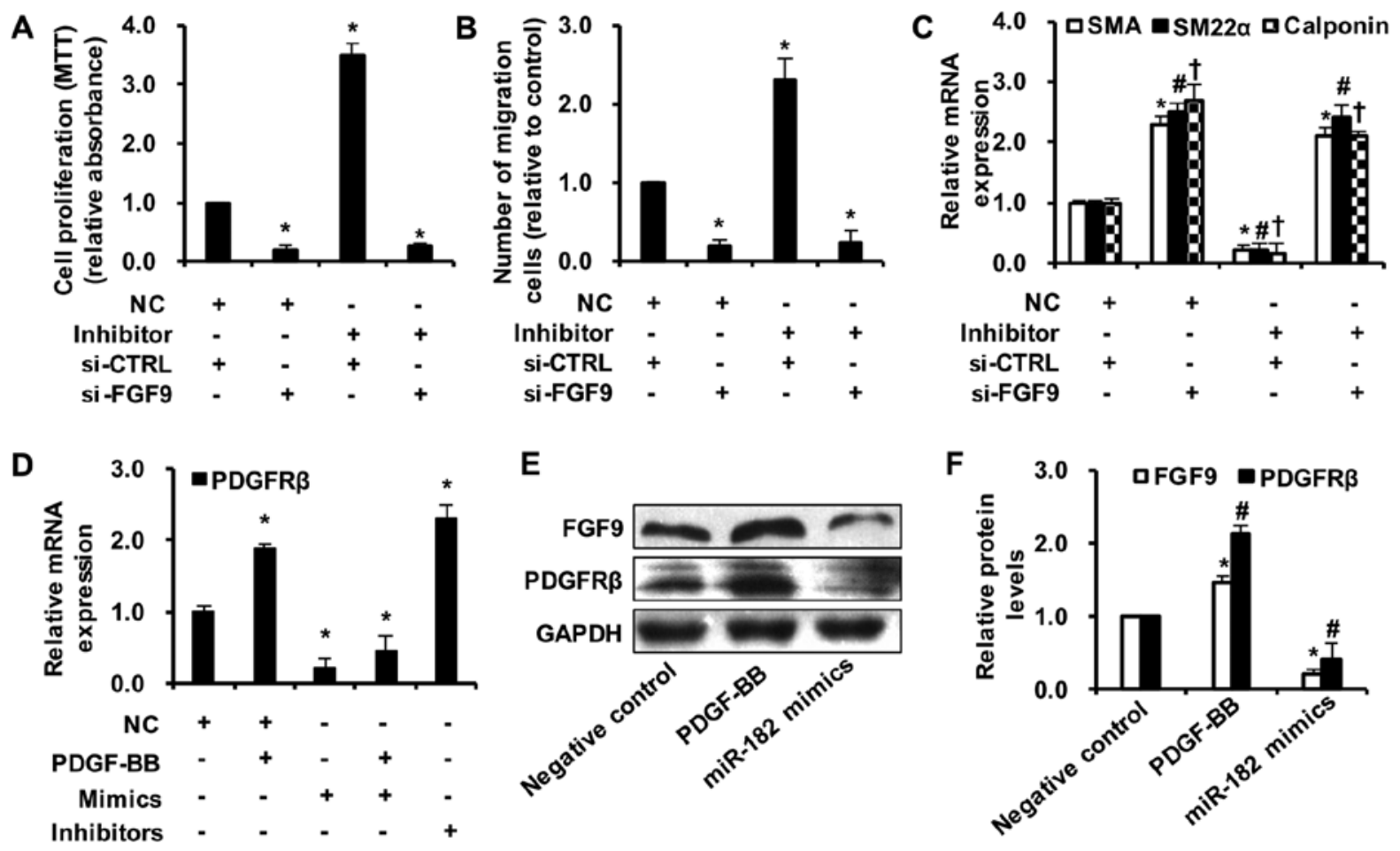

Figure 6. miR-182 mediates SMC phenotypic modulation via fibroblast growth factor 9 (FGF9)/platelet-derived growth factor receptor $\beta$ (PDGFR $\beta$ ) signaling. Smooth muscle cells (SMCs) were transfected with either miR-182 inhibitors $(80 \mathrm{nM})$, or with si-FGF9 (100 nM), or both. (A) miR-182 inhibitor transfection induced an increase in SMC proliferation; however, FGF9 downregulation inhibited miR-182 inhibitor-induced SMC proliferation, as determined by MTT assay (n=5). "p<0.05 vs. control. (B) FGF9 knockdown also interfered with the function of miR-182 inhibitors on SMC migration, as determined by scratch-wound healing assay $(n=5)$. ${ }^{*}<<0.05$ vs. control. (C) si-FGF9 transfection increased differentiated marker gene expression. However, miR-182 inhibitor transfection induced a decrease in SMC-specific gene expression. When si-FGF9 and miR-182 inhibitors were both transfected into SMCs, the expression of SMA, smooth muscle $22 \alpha$ (SM22 $\alpha$ ) and calponin was significantly increased, as determined by RT-qPCR ( $\mathrm{n}=5$ ). ${ }^{*} \mathrm{p}<0.05$ vs. the control group of SMA; ${ }^{*} \mathrm{p}<0.05$ vs. control of SM22 $\alpha ;{ }^{\dagger} p<0.05$ vs. control of calponin. (D) PDGF-BB treatment induced an increase in PDGFR $\beta$ expression, while this increase was suppressed by miR-182 mimic transfection and was enhanced by miR-182 inhibitor transfection ( $n=5)$. "p<0.05 vs. control. (E and F) The protein level of PDGFR $\beta$ was increased by PDGF-BB stimulation, but was decreased by miR-182 overexpression, as determined by (E) western blot analysis and (F) densitometric analysis $(\mathrm{n}=5)$ " $\mathrm{p}<0.05$ vs. negative control of FGF9; ${ }^{*} \mathrm{p}<0.05$ vs. negative control of PDGFR $\beta$.

microRNA.org database, we identified that FGF9 is one of the target genes of miR-182 across species. FGF9 combined with its cognate receptors is able to induce cell dedifferentiation and maturation (32). In addition, Yu et al proved that miR-182 inhibited FGF9 expression by binding to the 3'-UTR in rat Schwann cells using Luciferase reporter assay (13). These data suggest that FGF9 may be a direct target of miR-182 in rat vascular SMCs. This study revealed a dose-dependent decrease in FGF9 protein expression in miR-182 mimic-transfected SMCs. Accordingly, FGF9 is the target gene of miR-182 in rat vascular SMCs.

The downregulation of FGF9 or upregulation of miR-182 induced a similarly protective effect on the SMC phenotype and an inhibitory effect on proliferation and migration. miR-182 downregulation induced SMC differentiation, proliferation and migration; however, these processes were prevented by FGF9 knockdown. Moreover, the inhibition of FGF9 itself was able to suppress the dedifferentiation, proliferation and migration of rat SMCs. Although FGF9 was only one of the target genes, the results suggest that FGF9 is critical in the process of miR-182-mediated rat vascular SMC phenotypic modulation.

The activation of PDGFR $\beta$ is essential for the proliferation and migration of a variety of cells, and FGF9 has been implicated in upregulating of PDGFR $\beta$ expression $(16,18)$. Frontini et al reported that the FGF9-induced migration of cultured SMCs and neovascularization required both PDGFR $\beta$ and SHH, but not ERK1/2 (16). PDGF-AB or -BB binding with the PDGFR $\beta$ or the receptor overexpression results in the activation of PI3K/Akt, PLC $\gamma 1$ and MAPK pathways (33-36). PDGFR $\beta$ is a critical downstream target of FGF9 and an important signaling for FGF9-induced mural cell recruitment and vessel maturation (16). Thus, we hypothesized that the inhibition of SMC phenotypic modulation via the upregulation of miR-182 or the downregulation of FGF9 was related to PDGFR $\beta$ signaling. In this study, the expression of PDGFR $\beta$ was demonstrated to be markedly altered by regulating the expression of miR-182 and FGF9. Indeed, the downregulation of FGF9 decreased PDGFR $\beta$ expression, whereas the upregulation of FGF9 increased the PDGFR $\beta$ levels in rat vascular SMCs. In addition, PDGF-BB induced an increase in the expression of PDGFR $\beta$, which was significantly inhibited by miR-182 overexpression, but was upregulated following miR-182 knockdown. Thus, FGF9/PDGFR $\beta$ signaling is critical in the miR-182-mediated SMC phenotypic switch.

In conclusion, the present study demonstrated that the miR-182 level was altered markedly during the phenotypic transformation of rat vascular SMCs in vitro. The upregulation of miR-182 inhibited SMC dedifferentiation, proliferation and migration under both basal conditions and PDGF-BB stimulation. FGF9 was the critical target gene of miR-182 in the process of miR-182-mediated rat vascular SMC pheno- 
typic modulation. The downregulation of FGF9 increased SMC-specific contractile marker expression, and inhibited the proliferation and migration of SMCs via PDGFR $\beta$ signaling. This study suggests that miR-182 is a novel phenotypic marker, which modulates the differentiation, proliferation and migration of rat vascular SMCs via FGF9/PDGFR $\beta$ signaling. miR-182 may thus play a potential role in the diagnosis and treatment of vascular proliferative diseases.

\section{Acknowledgements}

The author Nana Dong has received grants from the Open Foundation in China's Ministry of Education (KF201401) and from the Graduate Student Innovation Foundation of Heilongjiang Province (YJSCX2012-217HLJ). This study was also supported by the National Key Project Foundation of China (no. 81330033). The authors would like to thank MedSci Editing for the revision of the manuscript.

\section{References}

1. Duband JL, Gimona M, Scatena M, Sartore S and Small JV: Calponin and SM 22 as differentiation markers of smooth muscle: Spatiotemporal distribution during avian embryonic development. Differentiation 55: 1-11, 1993.

2. Owens GK, Kumar MS and Wamhoff BR: Molecular regulation of vascular smooth muscle cell differentiation in development and disease. Physiol Rev 84: 767-801, 2004.

3. Davis-Dusenbery BN, Wu C, Hata A and Sessa WC: Micromanaging vascular smooth muscle cell differentiation and phenotypic modulation. Arterioscler Thromb Vasc Biol 31: 2370-2377, 2011.

4. Kim VN: MicroRNA biogenesis: Coordinated cropping and dicing. Nat Rev Mol Cell Biol 6: 376-385, 2005.

5. Niwa R and Slack FJ: The evolution of animal microRNA function. Curr Opin Genet Dev 17: 145-150, 2007.

6. Bartel DP: MicroRNAs: Genomics, biogenesis, mechanism, and function. Cell 116: 281-297, 2004

7. Hammond SM: RNAi, microRNAs, and human disease. Cancer Chemother Pharmacol 58 (Suppl 1): s63-s68, 2006.

8. Davis BN, Hilyard AC, Nguyen PH, Lagna G and Hata A: Induction of microRNA-221 by platelet-derived growth factor signaling is critical for modulation of vascular smooth muscle phenotype. J Biol Chem 284: 3728-3738, 2009.

9. Wang YS, Wang HY, Liao YC, Tsai PC, Chen KC, Cheng HY, Lin RT and Juo SH: MicroRNA-195 regulates vascular smooth muscle cell phenotype and prevents neointimal formation. Cardiovasc Res 95: 517-526, 2012.

10. Li P,Zhu N, Yi B, Wang N, Chen M, You X, Zhao X, Solomides CC, Qin Y and Sun J: MicroRNA-663 regulates human vascular smooth muscle cell phenotypic switch and vascular neointimal formation. Circ Res 113: 1117-1127, 2013.

11. Stenvold H, Donnem T, Andersen S, Al-Saad S, Busund LT and Bremnes RM: Stage and tissue-specific prognostic impact of miR-182 in NSCLC. BMC Cancer 14: 138, 2014.

12. Mihelich BL, Khramtsova EA, Arva N, Vaishnav A, Johnson DN, Giangreco AA, Martens-Uzunova E, Bagasra O, Kajdacsy-Balla A and Nonn L: miR-183-96-182 cluster is overexpressed in prostate tissue and regulates zinc homeostasis in prostate cells. J Biol Chem 286: 44503-44511, 2011.

13. Yu B, Qian T, Wang Y,Zhou S, Ding G, Ding F and Gu X: miR-182 inhibits Schwann cell proliferation and migration by targeting FGF9 and NTM, respectively at an early stage following sciatic nerve injury. Nucleic Acids Res 40: 10356-10365, 2012.

14. Naruo K, Seko C, Kuroshima K, Matsutani E, Sasada R, Kondo T and Kurokawa T: Novel secretory heparin-binding factors from human glioma cells (glia-activating factors) involved in glial cell growth. Purification and biological properties. J Biol Chem 268: 2857-2864, 1993.

15. Miyakawa K, Hatsuzawa K, Kurokawa T, Asada M, Kuroiwa T and Imamura T: A hydrophobic region locating at the center of fibroblast growth factor-9 is crucial for its secretion. J Biol Chem 274: 29352-29357, 1999.
16. Frontini MJ, Nong Z, Gros R, Drangova M, O'Neil C, Rahman MN, Akawi O, Yin H, Ellis CG and Pickering JG: Fibroblast growth factor 9 delivery during angiogenesis produces durable, vasoresponsive microvessels wrapped by smooth muscle cells. Nat Biotechnol 29: 421-427, 2011.

17. Andrae J, Gallini R, and Betsholtz C: Role of platelet-derived growth factors in physiology and medicine. Genes Dev 22: 1276-1312, 2008.

18. Hellström M, Kalén M, Lindahl P, Abramsson A and Betsholtz C: Role of PDGF-B and PDGFR-beta in recruitment of vascular smooth muscle cells and pericytes during embryonic blood vessel formation in the mouse. Development 126: 3047-3055, 1999.

19. Metz RP, Patterson JL and Wilson E: Vascular smooth muscle cells: Isolation, culture, and characterization. Methods Mol Biol 843: 169-176, 2012

20. Liu J, Wang Y, Du W, Liu W, Liu F, Zhang L, Zhang M, Hou M, Liu K, Zhang S and Yu B: Wnt1 inhibits hydrogen peroxideinduced apoptosis in mouse cardiac stem cells. PLoS One 8: e58883, 2013

21. Wang F, Zhong S, Zhang $\mathrm{H}$, Zhang $\mathrm{W}$, Zhang $\mathrm{H}$, Wu $\mathrm{X}$ and Chen B: Prognostic value of MicroRNA-182 in cancers: A meta-analysis. Dis Markers 2015: 482146, 2015.

22. Zhang W, Qian P, Zhang X, Zhang M, Wang H, Wu M, Kong X, Tan S, Ding K, Perry JK, et al: Autocrine/paracrine human growth hormone-stimulated MicroRNA 96-182-183 cluster promotes epithelial-mesenchymal transition and invasion in breast cancer. J Biol Chem 290: 13812-13829, 2015.

23. Li Y, Zhang D, Wang X, Yao X, Ye C, Zhang S, Wang H, Chang C, Xia H, Wang YC, et al: Hypoxia-inducible miR-182 enhances HIF1 $\alpha$ signaling via targeting PHD2 and FIH1 in prostate cancer. Sci Rep 5: 12495, 2015.

24. Du C, Weng X, Hu W, Lv Z, Xiao H, Ding C, Gyabaah OA, Xie H, Zhou L, Wu J and Zheng S: Hypoxia-inducible miR-182 promotes angiogenesis by targeting RASA1 in hepatocellular carcinoma. J Exp Clin Cancer Res 34: 67, 2015.

25. Chen L, Chu F, Cao Y, Shao J and Wang F: Serum miR-182 and miR-331-3p as diagnostic and prognostic markers in patients with hepatocellular carcinoma. Tumour Biol 36: 7439-7447, 2015.

26. Tang L, Chen F, Pang EJ, Zhang ZQ, Jin BW and Dong WF: MicroRNA-182 inhibits proliferation through targeting oncogenic ANUBL1 in gastric cancer. Oncol Rep 33: 1707-1716, 2015.

27. Sun Y, Fang R, Li C, Li L, Li F, Ye X and Chen H: Hsa-miR-182 suppresses lung tumorigenesis through downregulation of RGS17 expression in vitro. Biochem Biophys Res Commun 396: 501-507, 2010.

28. Moskwa P, Buffa FM, Pan Y, Panchakshari R, Gottipati P, Muschel RJ, Beech J, Kulshrestha R, Abdelmohsen K, Weinstock DM, et al: miR-182-mediated downregulation of BRCA1 impacts DNA repair and sensitivity to PARP inhibitors. Mol Cell 41: 210-220,2011.

29. Chamley-Campbell J, Campbell GR and Ross R: The smooth muscle cell in culture. Physiol Rev 59: 1-61, 1979.

30. Shanahan CM and Weissberg PL: Smooth muscle cell heterogeneity: Patterns of gene expression in vascular smooth muscle cells in vitro and in vivo. Arterioscler Thromb Vasc Biol 18: 333-338, 1998.

31. Kouri FM, Hurley LA, Daniel WL, Day ES, Hua Y, Hao L, Peng CY, Merkel TJ, Queisser MA, Ritner C, et al: miR-182 integrates apoptosis, growth, and differentiation programs in glioblastoma. Genes Dev 29: 732-745, 2015.

32. Cinaroglu A, Ozmen Y, Ozdemir A, Ozcan F, Ergorul C, Cayirlioglu P, Hicks D and Bugra K: Expression and possible function of fibroblast growth factor 9 (FGF9) and its cognate receptors FGFR2 and FGFR3 in postnatal and adult retina. J Neurosci Res 79: 329-339, 2005.

33. Heldin CH, Ostman A and Rönnstrand L: Signal transduction via platelet-derived growth factor receptors. Biochim Biophys Acta 1378: F79-F113, 1998.

34. Cospedal R, Abedi $\mathrm{H}$ and Zachary I: Platelet-derived growth factor-BB (PDGF-BB) regulation of migration and focal adhesion kinase phosphorylation in rabbit aortic vascular smooth muscle cells: Roles of phosphatidylinositol 3-kinase and mitogenactivated protein kinases. Cardiovasc Res 41: 708-721, 1999.

35. Song MC,Kim EC, Kim WJ and Kim TJ: Meso-dihydroguaiaretic acid inhibits rat aortic vascular smooth muscle cell proliferation by suppressing phosphorylation of platelet-derived growth factor receptor beta. Eur J Pharmacol 744: 36-41, 2014.

36. Caglayan E, Vantler M, Leppänen O, Gerhardt F, Mustafov L, Ten Freyhaus H, Kappert K, Odenthal M, Zimmermann WH, Tallquist MD and Rosenkranz S: Disruption of platelet-derived growth factor-dependent phosphatidylinositol 3-kinase and phospholipase $C \gamma 1$ activity abolishes vascular smooth muscle cell proliferation and migration and attenuates neointima formation in vivo. J Am Coll Cardiol 57: 2527-2538, 2011. 